\title{
Translating the oxidative stress hypothesis into the clinic: NOX versus NOS
}

\author{
Melanie E. Armitage • Kirstin Wingler • \\ Harald H. H. W. Schmidt • Mylinh La
}

Received: 19 August 2009 /Revised: 14 September 2009/Accepted: 17 September 2009/Published online: 16 October 2009

(C) Springer-Verlag 2009

\begin{abstract}
Cardiovascular diseases remain the leading cause of death in industrialised nations. Since the pathomechanisms of most cardiovascular diseases are not understood, the majority of therapeutic approaches are symptom-orientated. Knowing the molecular mechanism of disease would enable more targeted therapies. One postulated underlying mechanism of cardiovascular diseases is oxidative stress, i.e. the increased occurrence of reactive oxygen species such as superoxide. Oxidative stress leads to a dysfunction of vascular endothelium-dependent protective mechanisms. There is growing evidence that this scenario also involves impaired nitric oxide (NO)-cyclic GMP signalling. Out of a number of enzyme families that can produce reactive oxygen species, NADPH oxidases stand out, as they are the only enzymes whose sole purpose is to produce reactive oxygen species. This review focuses on the clinically validated targets of oxidative stress, NO synthase (NOS) and the NO receptor, soluble guanylate cyclase as well as the source of ROS, e.g. NADPH oxidases. We place recent knowledge in the function and regulation of these enzyme families into clinical perspective. For a comprehensive overview of the biology and pharmacology of oxidative stress and possible other sources and targets, we refer to other literature overviews.
\end{abstract}

Keywords Nitric oxide $\cdot$ Reactive oxygen species · Oxidative stress · sGC activators · sGC stimulators · NADPH oxidases (NOX) · Soluble guanylate cyclase

M. E. Armitage $\cdot$ K. Wingler $\cdot$ H. H. H. W. Schmidt $(\square) \cdot$ M. La Centre for Vascular Health, Department of Pharmacology, Monash University,

Building 13E, Wellington Rd, Clayton,

Victoria 3800, Australia

e-mail: harald.schmidt@med.monash.edu.au
In cardiovascular diseases (CVD) such as hypertension, atherosclerosis and chronic heart failure, endothelial dysfunction correlates with and can even predict long-term disease progression and outcome [1]. Endothelial dysfunction is defined as the impairment of endothelium-dependent relaxation. Whilst a number of factors contribute to endothelial dysfunction, compromised nitric oxide-cyclic GMP (NO-cGMP) signalling is a hallmark of this condition. Indeed, loss of vasodilatory and anti-platelet effects of NO may result in CVD initiation and progression. There is increasing evidence that pathophysiological production of reactive oxygen species (ROS) interferes with NO-cGMP signalling and may play a significant role in the development of endothelial dysfunction. At least three underlying mechanisms have been proposed (Fig. 1).

Firstly, ROS directly reduce the bioavailability of NO by chemical scavenging. NO reacts with excess superoxide, forming peroxynitrite $\left(\mathrm{ONOO}^{-}\right)$[2]. Secondly, ROS indirectly affect NO bioavailability by uncoupling endothelial NO synthase (eNOS). Mechanistically, this involves oxidation of the essential NOS redox-sensitive cofactor tetrahydrobiopterin $\left(\mathrm{BH}_{4}\right.$, see below) by ROS [1], which subsequently uncouples eNOS, which then produces superoxide instead of NO [3]. Thirdly, ROS alter both the expression and activity of the NO receptor, soluble guanylate cyclase (sGC). This mechanism involves oxidation of the sGC haem and subsequent haem loss [4, 5], ubiquitination of the empty haem pocket [6] and proteasomal degradation [7].

Several enzymes are capable of initiating this scenario, including xanthine oxidase, cyclooxygenase, lipoxygenase, uncoupled eNOS, cytochrome p450 and the mitochondrial electron chain. However, NADPH oxidases stand out as the major source of ROS as they are the only known enzyme family solely dedicated to ROS production. All other 


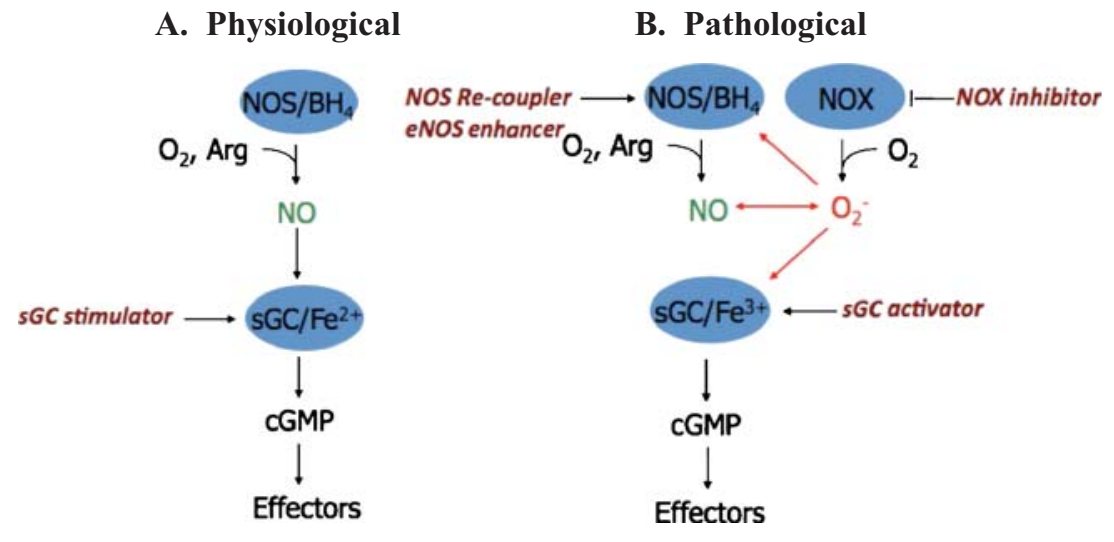

Fig. 1 The NO-sGC signalling pathway and potential drug targets under physiological and pathophysiological conditions. Under physiological conditions (A), NO, synthesised by NOS from L-arginine, activates soluble guanylate cyclase (sGC) leading to the formation of cGMP and downstream effector mechanisms. sGC stimulators enhance the sensitivity of $\mathrm{sGC}$ to low levels of bioavailable NO. Under pathological condition (B) such as oxidative stress, reactive oxygen species, e.g. superoxide $\left(\mathrm{O}_{2}{ }^{-}\right)$most likely derived from NADPH oxidases (NOX), affect the NO-sGC system by

known enzymes produce ROS as a by-product or as a consequence of a biochemical "accident". Importantly, of all known ROS targets, NOS and sGC show the clearest clinical relevance, as demonstrated by several ongoing drug development programmes in different clinical stages or even existing drugs in clinical practise. This brief review will therefore focus on the NADPH oxidase-NOS-governed fine balance of radicals. For further information into other sources of ROS, we refer the reader to several excellent reviews $[8,9]$.

\section{Enhancing endothelial NO synthesis}

NO is a ubiquitous signalling molecule with distinctive roles in diverse tissues and species [10]. It is synthesised either enzymatically from the amino acid L-arginine by NOS or generated non-enzymatically from nitrite under acidic conditions, e.g. in ischaemia/reperfusion [11]. Three isoforms of NOS exist: neuronal (nNOS/NOS1), inducible (iNOS/NOS2) and endothelial (eNOS/NOS3). Of these three isoforms, eNOS is the most relevant in cardiovascular system. eNOS is primarily present in endothelial cells, is constitutively expressed and synthesises NO for short time periods in response to receptor or physical stimulation. NO released by eNOS mediates several physiological and vasoprotective functions including the inhibition of vascular contraction and platelet activation. The activity of eNOS is dependent on the availability of $\mathrm{BH}_{4}$ and other cofactors.

Three approaches have been pursued to increase suboptimal levels of NO: the use of NO donors, stimulating three mechanisms: Superoxide scavenges NO; superoxide induces eNOS uncoupling, reducing NO production and enhanced superoxide production; superoxide oxidises the $\mathrm{NO}$ receptor, sGC, rendering it unresponsive to $\mathrm{NO}$ activation. Potential therapeutic strategies to diminish oxidative stress include the application of NADPH oxidase inhibitors, eNOS recoupler such as $\mathrm{BH}_{4}$ or eNOS enhancer (AVE 9488), and sGC stimulators of reduced $\left(\mathrm{Fe}^{2+}\right)$ or sGC activators of the oxidised $\left(\mathrm{Fe}^{3+}\right)$ and haem-free (apo-) sGC

eNOS activity by substrate supply or more recently by enhancing eNOS expression. NO donors have problematic pharmacokinetics, are prone to tolerance phenomena and lead to enhanced nitrative chemistry in the vascular wall [9]. The second approach, i.e. to stimulate NOS activity by co-factor $\left(\mathrm{BH}_{4}\right)$ or substrate (L-arginine) supplementation is mechanistically plausible, but the clinical efficacies of these approaches remain to be shown [12]. The bioavailability of orally supplied L-arginine may be limited by first-pass effects [13]. Moreover, L-arginine may act not only by enhancing substrate supply but also by displacing an inhibitory methylated L-arginine analogue, asymmetric dimethylarginine (ADMA), from the enzyme's substrate binding site [12]. In fact, plasma ADMA levels may represent one of the better markers of endothelial dysfunction and cardiovascular disease risk [12]. More recently, an eNOS enhancer, AVE9488, was introduced as a third approach to overcome insufficient NO synthesis by upregulating eNOS expression. AVE9488 protects against ischaemia/reperfusion injury in animal models, but the mechanism is not fully understood [14]. However, one foreseeable risk is the presence of oxidative stress as additionally expressed eNOS may become uncoupled, which can exacerbate the problem [3]. Nevertheless, the potential clinical benefits of eNOS enhancers certainly provide a new approach to treat CVD.

Interestingly, some pathological vascular conditions may be linked with excessive rather than reduced NO production. Stroke-induced neuronal death appears in part to be caused by excessive nNOS activation [15]. Similarly, unwarranted upregulation of iNOS causes refractory hypo- 
tension in septic shock [16]. Early studies showed that inhibitors of NOS were effective in normalising blood pressure in different animal models of sepsis, but attempts to translate these findings into an effective clinical therapy were disappointing to date. A multi-centre phase III study of 800 patients with a NOS inhibitor failed to show any benefit and had to be terminated early due to a high mortality rate [16]. Given that NO is a mediator of many essential physiological functions, it is not surprising that global inhibition of NOS has adverse effects. Therefore, selective iNOS inhibition reducing local - as opposed to a globalNO production may have more favourable effects. Recently, new selective iNOS inhibitors have become available. Nevertheless, their efficacy and safety in the clinic remain to be determined [17]. Interestingly, a recent provocative hypothesis proposed that NO is rather protective in sepsis. Indeed, enhancing NO formation via exogenous nitrite enhanced survival in a sepsis model [18]. Thus, we may see soon a dogma shift on the role of NO in sepsis, whilst the negative role in stroke seems unchallenged; therefore, stroke may be an indication for NOS inhibition.

\section{Potentiating NO via sGC stimulators}

Many physiological functions of NO are mediated through its primary receptor, sGC. sGC is a heterodimeric protein consisting of a homologous $\alpha$ and a haem-containing $\beta$ sub-unit. The haem group is located on the axial ligand histidine $^{105}$ of the $\beta$ subunit [5]. sGC activation by NO is haem-dependent and leads to the conversion of GTP to cGMP, which modulates the activity of several downstream effector molecules including protein kinases, phosphodiesterase (PDE) and ion channels [5]. According to the simplest model for sGC activation by NO, sGC is activated in two steps: NO first binds to the haem of sGC to form a six-coordinated $\mathrm{NO}-\mathrm{Fe}^{2+}$-Histidine ${ }^{105}$ intermediate complex, which is then rapidly converted to a penta-complex [19]. It is believed that the breakage of the haem-histidine ${ }^{105}$ bond on the $\beta$ subunit leads to the full activation of the enzyme [5].

Pharmacological activation of sGC has been employed for more than a century in the form of organic nitrates for the treatment of angina, acute and chronic heart failure. More recently, inhaled NO is used in acute respiratory distress syndrome and congenital heart defects. However, their clinical application is limited due to the development of tolerance from chronic administration and the route of administration, respectively [20]. Recently, it was discovered that $\mathrm{sGC}$ has an allosteric binding site where so-called sGC stimulators bind and potentiate sGC activation by NO.

Given the shortcomings of organic nitrates, the first report of a direct, NO-independent stimulator of sGC,
YC-1, in 1994 raised much interest [21]. In 2001, Bayer Healthcare AG reported a new class of sGC stimulators, with BAY 41-2272 as prototype [22]. sGC stimulators require an intact and functional prosthetic haem moiety in sGC and act synergistically with NO. In comparison to YC-1, BAY 41-2272 is a more potent sGC stimulator, is orally active and has no or less PDE-inhibiting properties [22]. Mechanistically, sGC stimulators increase the half-life of the nitrosyl-haem complex [5]. Despite their effectiveness in a variety of animal models of CVD, stimulators such as BAY 41-2272 and BAY 41-8543 showed unfavourable drug metabolism and pharmacokinetic (DMPK) properties. More recently another stimulator, Riociguat (BAY 63-2521), was found to have an improved DMPK profile. It has beneficial effects on pulmonary haemodynamics and exercise capacity in patients with pulmonary hypertension. Riociguat is currently in phase III clinical development for the oral treatment of pulmonary hypertension [23]. However, both enhanced NO synthesis, NO donors and sGC stimulators fail when the sGC haem is oxidatively damaged and lost. Here, a different class of sGC-modulating drug becomes important, sGC activators.

\section{Replacing NO by sGC activators}

sGC activators helped to elucidate a previously unrecognised alternative pathway to generate cGMP from sGC beyond the binding of $\mathrm{NO}$ to the $\mathrm{Fe}^{2+}$ haem of the $\mathrm{sGC}$ $\beta$ subunit. One key observation was the existence of a haem-oxidised sGC (ox-sGC) and even haem-free sGC (apo-sGC) [5]. It is now clear that ox-/apo-sGC is ubiquitously present in cells and tissues [5, 24]. It has been speculated that the oxidised form is elevated in CVD associated with increased levels of ROS [5].

In contrast to sGC stimulators which synergize with NO, BAY 58-2667 showed additive effects when combined with NO donors [5]. Further investigations revealed that NO and BAY 58-2667 activate sGC independently. It was proposed that BAY 58-2667 activates sGC by replacing the prosthetic haem or - in the case of the haem-free enzyme - by directly occupying the orphaned haem-pocket. At this year's 4th International Conference on cGMP, the crystal structure of a domain similar to that of the $\mathrm{N}$ terminus of $\mathrm{sGC} \beta$ and its interaction with BAY 58-2667 was reported. It was shown that BAY 58-2667 can indeed displace the haem by occupying the pocket and thus mimicking the interaction of haem with sGC [25]. More recently, BAY 58-2667 has demonstrated its efficacy in a proof-of-concept phase IIb clinical study in heart failure patients, reducing pre- and post-load and increasing cardiac output from baseline [26]. Another sGC activator, HMR1766 (Sanofi-Aventis) is 
currently in a phase II clinical study for treatment of neuropathic pain.

In summary, the physiological existence of haem-free apo-sGC can no longer be rejected. This raises questions about the ratio of haem-free to oxidised $\left(\mathrm{Fe}^{3+}\right)$ to reduced $\left(\mathrm{Fe}^{2+}\right)$ sGC in physiological and pathophysiological conditions, and why apo-sGC seems to be more prevalent under disease conditions. Given that many pathological conditions are associated with oxidative stress, the link between ROS and different redox states of sGC needs further elucidation.

Having described three validated and clinically relevant mechanisms by which ROS affect NOS and cGMP signalling and several pharmacologic approaches to reverse these, a more rational approach is to prevent oxidative damage occurring in the first place by identifying and targeting the major sources of ROS, e.g. NADPH oxidases.

\section{The relevant sources of ROS}

ROS include free radicals such as the superoxide $\left(\mathrm{O}_{2}{ }^{-}\right)$, the hydroxyl radical $(\mathrm{HO})$ and non-radical species such as hydrogen peroxide $\left(\mathrm{H}_{2} \mathrm{O}_{2}\right)$, hyperchlorous acid $(\mathrm{HClO})$ and peroxynitrite. ROS contribute to the homeostasis of a number of physiological systems including the respiratory, immune and neurological system [27]. Under physiological conditions, antioxidant defence enzymes scavenge and detoxify excess ROS. Endogenous antioxidants include superoxide dismutase, which dismutates superoxide into $\mathrm{H}_{2} \mathrm{O}_{2}$, as well as catalase and peroxidases, which convert $\mathrm{H}_{2} \mathrm{O}_{2}$ into water or other metabolites. Oxidative stress results from an imbalance between ROS generation and ROS inactivation. This may well be a highly localised subcellular event, as opposed to a systemic phenomenon.

Decreased inactivation of ROS appears to be a less common cause for oxidative stress [28] when compared to an increase in ROS generation. Thus, inhibiting enhanced ROS production is a promising strategy to restore endothelial function and to manage CVD. Of all the potential sources of ROS, NADPH oxidases are the only known enzyme family that has ROS as their sole enzymatic product [29]. NADPH oxidase was first identified in phagocytic cells as the enzyme responsible for the respiratory burst [30]. It later became apparent that these enzymes are also expressed in a variety of non-phagocytic cells, including all cells in the blood vessel wall [31], suggesting other functions besides host defence [9]. NADPH oxidases are enzyme complexes primarily distinguished by the membrane spanning catalytic NOX sub-unit that transfers electrons from NADPH to molecular oxygen. Five mammalian members of the NOX family have been identified (NOX1-5) [27] with NOX1, 2, 4 and 5 being expressed in the blood vessel wall. Importantly, NOX5 is not expressed in rodents, which may turn out to be a previously unrecognised limitation of mouse and rat models of oxidative stress. NOX isoforms rely to varying degrees on the association of regulatory proteins. NOX1and NOX2-containing isoforms require the association of a smaller membrane protein, p22phox, an "organiser" protein (p47phox or NOXO1), an "activator" protein (p67phox or NOXA1) and a small GTPase, Rac1 [9]. NOX4 may only require the association of p22phox, whilst NOX5 appears to function independently of any associated proteins but is regulated by $\left[\mathrm{Ca}^{2+}\right]$ [9]. NADPH oxidase activity in vascular cells is acutely increased by various pathological stimuli, such as angiotensin II, endothelin-1, growth factors, cytokines, metabolic factors (e.g. advanced glycation end products), oxidised LDL, mechanical forces and ischaemia-related stimuli [32]. For a more comprehensive overview on the molecular biology and regulation of NADPH oxidases, we refer to these reviews [9, 27, 29, 33-36].

Vascular NADPH oxidase-dependent superoxide production appears to be a driving force in the development of endothelial dysfunction in several animal models of CVD $[1,9]$. In coronary arteries of patients with CAD, NADPH oxidase activity is increased [37,38], as is expression of NOX5 [39]. In patients with diabetes, superoxide produced by NADPH oxidases and uncoupled eNOS significantly contributes to the endothelial dysfunction of arteries from these patients [40]. Nevertheless, the exact structure, regulation, distribution and patho-physiological role of the individual human NOX isoforms are only being unravelled.

\section{How to prevent or treat oxidative stress?}

As ROS are derived from a number of sources, it was deemed feasible to readjust the balance of ROS production and detoxification by supplementing antioxidants. However, clinical trials testing this hypothesis showed little benefits in reducing cardiovascular events or mortality; some of the treatments even caused harm [41]. Indeed, in vivo detoxification of increased ROS by antioxidant supplementation may be impossible due to the poor bioavailability of the antioxidants at the correct subcellular localisation and at the optimal time point. Antioxidants may also promote new radical chain reactions initiated by their oxidised forms. Importantly, the global removal of ROS may result in unwanted effects since ROS also regulate important physiological functions. Therefore, the current strategy is to identify and target the major sources of ROS, including NADPH oxidases.

A major constraint in NADPH oxidase research and translation is the lack of specific inhibitors. All commonly 
used compounds, including apocynin and diphenyleneiodonium, are unspecific [42] (Wind et al., unpublished observations), and there are no specific NOX inhibitors commercially available. The novel NADPH oxidase inhibitor, VAS2870, inhibits PDGF-stimulated cell migration and NADPH activity in vascular smooth muscle cells [43] as well as oxidised LDL-stimulated ROS formation in endothelial cells [44]. However, its mechanism of action and selectivity for different NOX isoforms is unknown. In vivo experiments with a small peptide inhibitor, gp91ds-tat, which interferes with the interaction between NOX2 and the regulator $\mathrm{p} 47 \mathrm{phox}$, had promising results, e.g. it reduced angioplasty-induced superoxide and neointimal hyperplasia of rat carotid artery [45]. As individual NOX isoforms are regulated by different subunits, targeting these protein-protein interactions is one strategy for the development of isoform-specific NOX inhibitors. For more details on NADPH oxidase inhibitors and their limitations, we refer to other published reviews [32, 46-48]. Once specific inhibitors become available, their potential to manage CVD by preventing ROS formation can be fully explored. This concept may also be applicable to other chronic diseases associated with oxidative stress.

\section{Conclusions}

The dissection of the oxidative stress hypothesis into a detailed identification of clinically relevant enzymes targeted by ROS offers for the first time a more mechanistic approach to CVD therapy. eNOS recouplers/enhancers as well as sGC stimulators/activators target specific oxidative damage, which currently can only be detected at a functional level. Nevertheless, clinical efficacy and proof-of-concept animal models promise a far more positive outcome than for previous antioxidant approaches. Thinking foward, the NADPH oxidase field represents an uncharted territory in the management of CVD. We believe that the development of NOX isoform selective inhibitors in the next decade will reveal the clinical relevance of this enzyme family.

Twenty years after its discovery [49], NO has come of age. It may be not so much NO synthesis itself but the signalling and oxidative events surrounding this unusual molecule that may produce the therapeutic outcomes that have been postulated for this highly protective vascular pathway and for CVD management.

\section{References}

1. Schulz E, Jansen T, Wenzel P, Daiber A, Munzel T (2008) Nitric oxide, tetrahydrobiopterin, oxidative stress, and endothelial dysfunction in hypertension. Antioxid Redox Signal 10:1115-1126
2. Rubanyi GM, Vanhoutte PM (1986) Superoxide anions and hyperoxia inactivate endothelium-derived relaxing factor. Am J Physiol 250:H822-H827

3. Mollnau H, Wendt M, Szocs K, Lassegue B, Schulz E, Oelze M, Li H, Bodenschatz M, August M, Kleschyov AL, Tsilimingas N, Walter U, Forstermann U, Meinertz T, Griendling K, Munzel T (2002) Effects of angiotensin II infusion on the expression and function of $\mathrm{NAD}(\mathrm{P}) \mathrm{H}$ oxidase and components of nitric oxide/cGMP signaling. Circ Res 90:E58-E65

4. Stasch JP, Schmidt PM, Nedvetsky PI, Nedvetskaya TY, SA H, Meurer S, Deile M, Taye A, Knorr A, Lapp H, Muller H, Turgay Y, Rothkegel C, Tersteegen A, Kemp-Harper B, Muller-Esterl W, Schmidt HH (2006) Targeting the heme-oxidized nitric oxide receptor for selective vasodilatation of diseased blood vessels. J Clin Invest 116:2552-2561

5. Evgenov OV, Pacher P, Schmidt PM, Hasko G, Schmidt HH, Stasch JP (2006) NO-independent stimulators and activators of soluble guanylate cyclase: discovery and therapeutic potential. Nat Rev Drug Discov 5:755-768

6. Meurer S, Pioch S, Pabst T, Opitz N, Schmidt PM, Beckhaus T, Wagner K, Matt S, Gegenbauer K, Geschka S, Karas M, Stasch JP, Schmidt HH, Muller-Esterl W (2009) Nitric oxide-independent vasodilator rescues heme-oxidized soluble guanylate cyclase from proteasomal degradation. Circ Res 105:33-41

7. Nedvetsky PI, Meurer S, Opitz N, Nedvetskaya TY, Muller H, Schmidt HH (2008) Heat shock protein 90 regulates stabilization rather than activation of soluble guanylate cyclase. FEBS Lett 582:327-331

8. Papaharalambus CA, Griendling KK (2007) Basic mechanisms of oxidative stress and reactive oxygen species in cardiovascular injury. Trends Cardiovasc Med 17:48-54

9. Bedard K, Krause KH (2007) The NOX family of ROS-generating NADPH oxidases: physiology and pathophysiology. Physiol Rev 87:245-313

10. Moncada S, Higgs EA (2006) Nitric oxide and the vascular endothelium. Handb Exp Pharmacol 176(Pt 1):213-254

11. Schulz R, Kelm M, Heusch G (2004) Nitric oxide in myocardial ischemia/reperfusion injury. Cardiovasc Res 61:402-413

12. Boger RH (2008) L-Arginine therapy in cardiovascular pathologies: beneficial or dangerous? Curr Opin Clin Nutr Metab Care 11:55-61

13. Morris SM Jr (2004) Enzymes of arginine metabolism. J Nutr 134:2743S-2747S discussion 2765S-2767S

14. Katusic ZS, d'Uscio LV, Nath KA (2009) Vascular protection by tetrahydrobiopterin: progress and therapeutic prospects. Trends Pharmacol Sci 30:48-54

15. Eliasson MJ, Huang Z, Ferrante RJ, Sasamata M, Molliver ME, Snyder SH, Moskowitz MA (1999) Neuronal nitric oxide synthase activation and peroxynitrite formation in ischemic stroke linked to neural damage. J Neurosci 19:5910-5918

16. Cobb JP (2001) Nitric oxide synthase inhibition as therapy for sepsis: a decade of promise. Surg Infect (Larchmt) 2:93-100 discussion 100-101

17. Tinker AC, Wallace AV (2006) Selective inhibitors of inducible nitric oxide synthase: potential agents for the treatment of inflammatory diseases? Curr Top Med Chem 6:77-92

18. Brouckaert P, Cauwels A, Thoonen R, Buys E, Bloch K, Sips P, Ichinose F, Rogge E, Simmegeers S, Van de Voorde J, Lefebvre R (2009) Phenotypes of sGC mutant mice in basic conditions, disease and shock. BMC Pharmacol 9(Suppl 1):S5

19. Stone JR, Sands RH, Dunham WR, Marletta MA (1996) Spectral and ligand-binding properties of an unusual hemoprotein, the ferric form of soluble guanylate cyclase. Biochemistry 35:3258-3262

20. Daiber A, Wenzel P, Oelze M, Munzel T (2008) New insights into bioactivation of organic nitrates, nitrate tolerance and crosstolerance. Clin Res Cardiol 97:12-20 
21. Ko FN, Wu CC, Kuo SC, Lee FY, Teng CM (1994) YC-1, a novel activator of platelet guanylate cyclase. Blood 84:4226-4233

22. Stasch JP, Becker EM, Alonso-Alija C, Apeler H, Dembowsky K, Feurer A, Gerzer R, Minuth T, Perzborn E, Pleiss U, Schroder H, Schroeder W, Stahl E, Steinke W, Straub A, Schramm M (2001) NO-independent regulatory site on soluble guanylate cyclase. Nature 410:212-215

23. Mittendorf J, Weigand S, Alonso-Alija C, Bischoff E, Feurer A, Gerisch M, Kern A, Knorr A, Lang D, Muenter K, Radtke M, Schirok H, Schlemmer KH, Stahl E, Straub A, Wunder F, Stasch JP (2009) Discovery of riociguat (BAY 63-2521): a potent, oral stimulator of soluble guanylate cyclase for the treatment of pulmonary hypertension. Chem Med Chem 4:853-865

24. Stasch JP, Schmidt P, Alonso-Alija C, Apeler H, Dembowsky K, Haerter M, Heil M, Minuth T, Perzborn E, Pleiss U, Schramm M, Schroeder W, Schroder H, Stahl E, Steinke W, Wunder F (2002) NO- and haem-independent activation of soluble guanylyl cyclase: molecular basis and cardiovascular implications of a new pharmacological principle. Br J Pharmacol 136:773-783

25. van Den Akker F, Ma X, Martin F, Pattanaik P, Padayatti P, Warman M, Beuve A (2009) Structural insights into sGC. BMC Pharmacol 9(Suppl 1):S42

26. Lapp H, Mitrovic V, Franz N, Heuer H, Buerke M, Wolfertz J, Mueck W, Unger S, Wensing G, Frey R (2009) Cinaciguat (BAY 58-2667) improves cardiopulmonary hemodynamics in patients with acute decompensated heart failure. Circulation 119:2781-2788

27. Lambeth JD (2007) NOX enzymes, ROS, and chronic disease: an example of antagonistic pleiotropy. Free Radic Biol Med 43:332347

28. Heistad DD, Wakisaka Y, Miller J, Chu Y, Pena-Silva R (2009) Novel aspects of oxidative stress in cardiovascular diseases. Circ J 73:201-207

29. Opitz N, Drummond GR, Selemidis S, Meurer S, Schmidt HH (2007) The 'A's and 'O's of NADPH oxidase regulation: a commentary on "subcellular localization and function of alternatively spliced Noxo1 isoforms". Free Radic Biol Med 42:175-179

30. Babior BM, Kipnes RS, Curnutte JT (1973) Biological defense mechanisms. The production by leukocytes of superoxide, a potential bactericidal agent. J Clin Invest 52:741-744

31. Griendling KK, Minieri CA, Ollerenshaw JD, Alexander RW (1994) Angiotensin II stimulates NADH and NADPH oxidase activity in cultured vascular smooth muscle cells. Circ Res 74:1141-1148

32. Cave A (2009) Selective targeting of NADPH oxidase for cardiovascular protection. Curr Opin Pharmacol 9:208-213

33. Sumimoto H, Miyano K, Takeya R (2005) Molecular composition and regulation of the Nox family NAD $(\mathrm{P}) \mathrm{H}$ oxidases. Biochem Biophys Res Commun 338:677-686

34. Bitar MS, Wahid S, Mustafa S, Al-Saleh E, Dhaunsi GS, Al-Mulla F (2005) Nitric oxide dynamics and endothelial dysfunction in type II model of genetic diabetes. Eur J Pharmacol 511:53-64

35. Brown DI, Griendling KK (2009) Nox proteins in signal transduction. Free Radic Biol Med.. doi:S0891-5849(09)00442-0[pii] 10.1016/j.freeradbiomed.2009.07.023

36. Brandes RP, Schroder K (2008) Differential vascular functions of Nox family NADPH oxidases. Curr Opin Lipidol 19:513-518
37. Spiekermann S, Landmesser U, Dikalov S, Bredt M, Gamez G, Tatge H, Reepschlager N, Hornig B, Drexler H, Harrison DG (2003) Electron spin resonance characterization of vascular xanthine and $\mathrm{NAD}(\mathrm{P}) \mathrm{H}$ oxidase activity in patients with coronary artery disease: relation to endothelium-dependent vasodilation. Circulation 107:1383-1389

38. Guzik TJ, Sadowski J, Guzik B, Jopek A, Kapelak B, Przybylowski P, Wierzbicki K, Korbut R, Harrison DG, Channon KM (2006) Coronary artery superoxide production and nox isoform expression in human coronary artery disease. Arterioscler Thromb Vasc Biol 26:333-339

39. Guzik TJ, Chen W, Gongora MC, Guzik B, Lob HE, Mangalat D, Hoch N, Dikalov S, Rudzinski P, Kapelak B, Sadowski J, Harrison DG (2008) Calcium-dependent NOX5 nicotinamide adenine dinucleotide phosphate oxidase contributes to vascular oxidative stress in human coronary artery disease. J Am Coll Cardiol 52:1803-1809

40. Guzik TJ, Mussa S, Gastaldi D, Sadowski J, Ratnatunga C, Pillai $\mathrm{R}$, Channon KM (2002) Mechanisms of increased vascular superoxide production in human diabetes mellitus: role of NAD (P)H oxidase and endothelial nitric oxide synthase. Circulation 105:1656-1662

41. Dotan Y, Pinchuk I, Lichtenberg D, Leshno M (2009) Decision analysis supports the paradigm that indiscriminate supplementation of vitamin E does more harm than good. Arterioscler Thromb Vasc Biol 29:1304-1309

42. Heumuller S, Wind S, Barbosa-Sicard E, Schmidt HH, Busse R, Schroder K, Brandes RP (2008) Apocynin is not an inhibitor of vascular NADPH oxidases but an antioxidant. Hypertension $51: 211-217$

43. ten Freyhaus H, Huntgeburth M, Wingler K, Schnitker J, Baumer AT, Vantler M, Bekhite MM, Wartenberg M, Sauer H, Rosenkranz S (2006) Novel Nox inhibitor VAS2870 attenuates PDGFdependent smooth muscle cell chemotaxis, but not proliferation. Cardiovasc Res 71:331-341

44. Stielow C, Catar RA, Muller G, Wingler K, Scheurer P, Schmidt $\mathrm{HH}$, Morawietz H (2006) Novel Nox inhibitor of oxLDL-induced reactive oxygen species formation in human endothelial cells. Biochem Biophys Res Commun 344:200-205

45. Jacobson GM, Dourron HM, Liu J, Carretero OA, Reddy DJ, Andrzejewski T, Pagano PJ (2003) Novel NAD(P)H oxidase inhibitor suppresses angioplasty-induced superoxide and neointimal hyperplasia of rat carotid artery. Circ Res 92:637-643

46. Williams HC, Griendling KK (2007) NADPH oxidase inhibitors: new antihypertensive agents? J Cardiovasc Pharmacol 50:9-16

47. Lambeth JD, Krause KH, Clark RA (2008) NOX enzymes as novel targets for drug development. Semin Immunopathol 30:339-363

48. Selemidis S, Sobey CG, Wingler K, Schmidt HH, Drummond GR (2008) NADPH oxidases in the vasculature: molecular features, roles in disease and pharmacological inhibition. Pharmacol Ther 120:254-291

49. Palmer RM, Ferrige AG, Moncada S (1987) Nitric oxide release accounts for the biological activity of endothelium-derived relaxing factor. Nature 327:524-526 